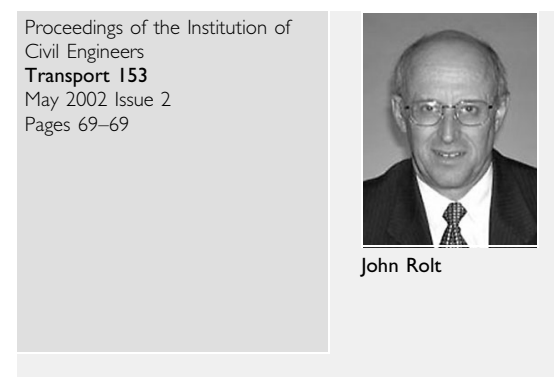

\title{
Editorial
}

\section{J. Rolt}

Disturbances from noise and vibration are becoming an increasingly important consideration for transport professionals involved in planning, design, construction, maintenance and in operations. Noise from transport affects large numbers of people and is, arguably, the most pervasive of all the environmental disbenefits associated with transport. Previous surveys indicate that in OECD countries alone, about 20 percent of the population, or nearly 135 million people, are exposed to noise from transport at levels that most authorities admit is unacceptably high. Increasingly stringent planning and assessment processes and enhanced public expectations place growing pressures on engineers and developers. Often they must demonstrate that there will be no unacceptable impacts from noise or vibration from new works.

Expected trends in noise levels over the next few years suggest that some improvements will be made. Road vehicles and aircrafts are subject to stringent limits on noise emission and this will continue to help reduce noise levels as new generation vehicles enter the traffic stream. Legislation on tyre noise and improvements in the design of both tyres and road surfaces will help to reduce road traffic noise on high-speed roads.

As well as reducing noise and vibration levels at source, improvements are also being made in reducing impact through consideration of the propagation path. Techniques include better design of acoustic barriers, making the best use of landscape and land use, and a greater understanding of the influence of meteorological conditions. Improvements in prediction modelling will help us to identify problems from noise and vibration at an early stage in the design process and will assist in creating solutions through a variety of techniques.

The effect of noise on people is also the subject of research. This ranges from mild annoyance through to disturbances that influence behaviour and activities. There is also a growing concern that noise at levels commonly experienced in the community can also have an adverse effect on health.

Vibration and noise from transport and transport engineering is, therefore, an important and multifaceted topic and we still have much to learn if we are to achieve acceptable levels in the future. This special edition of Transport covers a balance of topics. It includes papers on the measurement of noise itself, papers on controlling noise and vibration at source, a review article on acoustic barriers, research papers that enhance our understanding of both noise generation and noise propagation, and some case studies highlighting issues and best practice. Many of the topics are common to road, rail and air transport. Thus I am sure that this edition includes much that should be of interest to all readers. 